\title{
¿Uno o varios tipos de gobernanza? Más allá de la gobernanza como moda: la prueba del tránsito organizacional
}

\author{
David Arellano GaUlT \\ Centro de Investigación y Docencia Económica (CIDE) \\ david.arellano@cide.edu \\ José SÁnChez CetinA \\ Profesor Asociado del CIDE \\ jose.sanchez@cide.edu \\ Bartolo Retana Soto \\ Investigador Independiente. Mtro. en Administración y Políticas Públicas por el CIDE
}

Recibido: $23 / 10 / 2014$

Aceptado: 03/12/2014

\begin{abstract}
Resumen
El concepto de gobernanza parece necesitado de una discusión más amplia y escéptica: es un concepto en busca de contenido más empírico y menos normativo. En este artículo se presentan dos discusiones enlazadas: una que muestra al concepto de gobernanza muchas veces vacío de contenido, demasiado normativo en muchos sentidos, y otra que discute los límites desde la teoría de la organización de un concepto sobre idealizado de gobernanza democrática. Particularmente, en este último punto, se enfatiza el hecho organizacional de la acción de actores plurales en marcos de poder desigual como un reto a una perspectiva que asume al proceso de gobernanza más horizontal como deseable y efectivo en cualquier circunstancia o contexto. Se discute la falla de concepción que lleva a asumir que las redes sustituirán a las organizaciones y por tanto donde estos agentes organizacionales plurales y conflictivos pasan a segundo plano. Se concluye con un llamado a elevar el rango de investigación sobre gobernanza a una lógica más empírica, escéptica y que pruebe, más que asuma, las ventajas y desventajas de distintas formas de gobernanza.
\end{abstract}

Palabras clave: Gobernanza, teoría de la organización, nueva gestión pública, administración pública, teoría de redes.

\section{Governance: one size fits all? Beyond the fashion of governance, the test of «organizational transit»}

\begin{abstract}
The concept of governance seems to urgently require a broader and more skeptical view: it is currently a concept in need to become less normative and more empirical. In this paper we present two linked discussions: one on the need to discuss governance in a less normative way and more in an empirical one; and second on the limits of the concept from certain discussion coming from organizational theory. The main idea is to emphasize the importance of considering the omnipresence of organizational actors that are actually a set of pluralistic differentiated agents acting on social settings where power and conflict are indisputable aspects of their interaction. This is a challenge for a concept of governance that rests on the misunderstanding of organizations being substituted by networks. And also based on a misconception of the nature of a more horizontal way of governance where these organizational particularities seem to disappear. The conclusion support to improve the research on governance to a more empirical status, more skeptical in nature therefore, looking to test and prove both advantages and disadvantages of different types of governance structures.
\end{abstract}

Key words: governance, organization theory, new public management, public administration, network theory. 


\section{Introducción}

La democracia se ha convertido, pese a sus fallos y carencias (Rosanvallon, 2008: 2), en un valor discursivo y en una realidad social casi mundial. En muchos sentidos, es también un valor y una institución a la que, puede argüirse, se ha sobrecargado de expectativas. Se espera incluso que sea capaz de crear mecanismos efectivos y a la vez participativos para el abordaje de los problemas públicos en todo tipo de sociedades, prestando poca importancia en el fondo a las particularidades históricas y culturales a las que se enfrenta como mecanismo político en diversos países y regiones (Fukuyama, 1992).

Ahora bien, cuando la dinámica política real en una sociedad particular no genera tal promesa de participación y efectividad para la solución de los problemas públicos, muchos confían en que instrumentos tales como el «diseño institucional» (Goodin, 1996, Acemoglu y Robinson, 2012), las reformas política y administrativa (Banco Mundial, 1997) o, más recientemente, la gobernanza democrática (Aguilar, 2006) permitan resolver casi cualquier problema público o social. Particularmente, la gobernanza democrática se ha convertido en una tendencia, un discurso y una moda sobre la cual se espera que el aparato gubernamental y su acción se transformen, «modernicen» y entablen dinámicas de participación y coordinación social, siendo por ello (y no pese a ello) más efectivas y eficientes.

La literatura contemporánea que cuestiona los discursos y esperanzas racionalistas del «diseño institucional» de formas de gobernanza es creciente (Christensen y Laegreid, 2007; Brunsson, 2009; Hood, 1991, 2011; Arellano, 2002). En el caso de la gobernanza, pareciera entonces necesario hacer lo mismo que se ha realizado con relativo éxito respecto de otros discursos, como la nueva gestión pública: construir una visión crítica y escéptica como un primer paso. Comenzar, por ejemplo, con delinear un contenido más preciso al término de gobernanza (Grindle, 2010). Hay quienes incluso ya plantean que es un concepto francamente fastidioso (annoying, Perry, et al, 2014: 27). El concepto de gobernanza se ha convertido en una tendencia popular en muchos sentidos y valdría incluso la pena preguntarse cuándo en una sociedad democrática el gobierno ha sido la única solución, como repite con insistencia la retórica de la gobernanza. En realidad, resulta más sencillo encontrar experiencias donde la regla común ha sido desde siempre que los actores gubernamentales en una democracia han debido negociar, cooptar, discutir e involucrar a diversos actores políticos y económi$\cos ^{1}$.

Se ha popularizo en los últimos años un concepto de gobernanza que defiende un modelo multiparticipativo, descentralizado, con pluricentros de poder y autoridad. Gobernanza como técnica de «gobierno-más-allá-del-gobierno» pero, sobre todo,

${ }^{1}$ Para muestra, dos ejemplos: el famoso y muticitado caso expuesto por Selznick (1949) ya desde la década de los cuarenta, de la TVA. Y más recientemente el caso de la estación de autobuses de la ciudad de Aalborg, caso de inicios de la década de los 70 (expuesta a detalle en Flyvbjerg, 1991). Ambos muestran cómo, desde siempre en realidad, un gobierno democrático es sólo una parte de la pieza de la acción pública. 
como estilo de acción en una sociedad democrática plural. El punto de partida de este artículo es que, si bien dicha participación, decisión y coordinación parecen necesarias o pueden ser consideradas inevitables en una sociedad compleja, dicha dinámica no modifica de facto la lógica organizacional más profunda que tiene que crear las condiciones para la acción razonable y posible a la luz de procesos de poder diferenciado y actores plurales. De manera particular, el concepto de gobernanza democrática puede ser un discurso normativo interesante pero, en la práctica, un concepto con grandes limitaciones a la luz de la dinámica de la acción organizacional que se requiere para pasar de la definición de un problema público a la acción.

Pasar de la propuesta normativa a la acción implica una diferencia considerable pues, al final de cuentas, será en el contexto organizacional donde gran parte del diseño y de la implementación se cristalizarán, siendo las organizaciones espacios de poder articulados por personas heterogéneas comportándose en dinámicas conflictivas (Simon, 1991). La lógica organizacional muestra que en la práctica el diseño y la acción se relacionan (hay acciones sin diseño, diseño sin acciones y resultados que nadie diseñó ni buscó en la acción, Brunsson, 2007a). Que el diseño esté cimentado en mecanismos de gobernanza democrática no cambia los dilemas concretos de la acción, menos de la acción organizada. Tal vez, incluso, la transforme en un fenómeno aún más complejo e intrincado.

Las organizaciones son omnipresentes en la vida moderna y, en ese sentido, cualquier enfoque o propuesta de cambio social tendría que enfrentar los clásicos «efectos no deseados de la acción» tanto sociales como organizacionales. La idea del presente artículo es, justamente, construir a partir de lo que comprendemos de esta dinámica (sobre todo gubernamental, pero no exclusivamente) aquello que implica en concreto la lógica de la gobernanza en el terreno de la acción, más allá de su visión normativa. En otras palabras: la gobernanza parte de una idea normativa, a saber, la participación de muchos actores y grupos con diferentes pesos y perspectivas, creando una red de relaciones y decisiones en busca de una serie de objetivos relativamente compartidos. Las complejidades sociales y las dinámicas políticas que se generan por un esfuerzo de gobernanza más horizontal son, indudablemente, múltiples. En este artículo nos concentramos exclusivamente en la lógica organizacional gubernamental donde una gran parte de tales decisiones tendrán que llevarse a cabo e incluso constituirse como viables, legítimas y factibles. Probablemente lo que se encuentre redunde en que no sólo la lógica organizacional se vea afectada por la gobernanza, sino que la gobernanza misma tendría que ser transformada y entendida de manera diferente debido a la lógica organizacional.

De una manera más concreta, este artículo parte de la sospecha de que la gobernanza, entendida normativamente, tendría que ser adaptada en su discurso para transitar analíticamente de ser una solución general para casi todo problema a un instrumental alternativo consciente de sus limitaciones prácticas e incluso de las posibles consecuencias no deseadas que pueda generar. Vista de este modo, la gobernanza es una solución o instrumento político parcial y, por ende, no universal, no exento de generar efectos no deseados, por lo que debe ser analizada como una opción deseable y factible en algunas y no todas las ocasiones. Más aún, la gobernanza como instrumento será susceptible a fallas de implementación y sus resultados dependerán, en gran medida, de la forma concreta en que se diseñe, instaure y lleve a cabo. 
El artículo se estructura en tres secciones. En la primera se describe el diagnóstico del que parte la gobernanza, incorporando ya elementos críticos, así como los principales argumentos teóricos -el componente normativo- que la vincularían con la acción democrática. Presenta, además, un intento por comprender a la gobernanza desde un continuo con dos extremos: por un lado la gobernanza entendida de manera más simple y directa, como mecanismo (con pros y contras) y, en el otro extremo, la gobernanza «con adjetivos» que se ha convertido en una propuesta de cuño normativo. La segunda sección presenta tres zonas de tránsito en la modalidad de factores característicos de las organizaciones. El primero de estos factores se refiere a la lógica de la inclusión de actores distintos en dinámicas tanto cooperativas como competitivas, cuestión importante para evaluar si las redes organizacionales pueden garantizar o no la inclusión y la participación de los actores en el abordaje de problemas públicos. Posteriormente, se plantean posibles implicaciones relacionadas con algunos de los prerrequisitos de la acción democrática derivados de una toma de decisiones y acción organizacionales, configuradas muchas de las veces desde la irracionalidad de los actores y sus intenciones/acciones. Por último, se presentan algunos factores que permiten entender la capacidad de una red de organizaciones para adaptarse y mantener cierta dirección compatible con las necesidades de una democracia plural.

\section{La acción democrática desde la perspectiva de la gobernanza}

El concepto de gobernanza no es un monolito, sino un concepto «paraguas» que ha sido utilizado para cuestiones tan diversas que van desde un «gobierno sin gobierno» hasta un gobierno cuya responsabilidad sería el desarrollo mismo del sistema de redes organizacionales (Aguilar, 2006). El problema radica en que ha llegado a ser tan amplio y ambiguo que se le asocia ya como abarcador de innumerables cualidades: buen gobierno, transparencia, desarrollo económico, democracia y participación, sólo por mencionar algunas de ellas. Cabría preguntarse si al concepto de gobernanza no le ha pasado ya el mismo argumento que en su momento Simon (1947) utilizó para criticar la inutilidad de conceptos generales en la administración: la gobernanza ¿se ha convertido en un proverbio? En realidad, lo que puede asegurarse es que existe un número considerable de definiciones diferentes de gobernanza, con alcances y precisiones diversas, dependiendo del adjetivo que le acompañe.

En este artículo proponemos dos bloques de definiciones de la gobernanza en lo que, de hecho, es un continuo de variaciones conceptuales muy amplias. En un extremo colocaremos el concepto de gobernanza «a secas», entendiéndolo como instrumento o mecanismo general. Sin adjetivos, por decirlo de alguna manera. En el otro extremo se revisa el concepto de gobernanza democrática, «buena gobernanza»o gobernanza pluricentrista (asumiendo como similares estos términos, si bien pueden ser distintos en diversos aspectos). Este extremo normativo de la gobernanza le añade ciertos adjetivos que en un gran número de ocasiones intentan establecer el sesgo hacia lo que es deseable en la gobernanza. La finalidad de este continuo es clarificar el concepto para poder observar no sólo sus virtudes, sino también sus límites y problemáticas. En otras palabras, se trata de analizar a la gobernanza como un mecanismo en el 
sentido de Elster (2007): un factor causal que produce efectos que pueden variar ampliamente dependiendo de las circunstancias donde se aplica.

\subsection{Gobernanza «a secas»}

Partiremos del significado menos complejo del término. Esto es, antes de atribuirle virtudes, beneficios o limitaciones, la gobernanza puede entenderse como una estructuración de un proceso de toma de decisiones entre diversos actores, grupos u organizaciones. Este concepto clásico parece un buen lugar para iniciar dado que permite que conceptos genéricos como «gobernanza corporativa» o «gobernanza de la empresa» (Zingales, 2008) tengan sentido. De la misma manera, gobernanza ha sido utilizada en otros ámbitos de las organizaciones: gobernanza de proyectos (Garland, 2009) o de portafolios (Hanford, 2006), por ejemplo.

Visto en este sentido general, el término gobernanza «determina quién tiene el poder, quién toma las decisiones, cómo intervienen en el proceso otros jugadores y cómo se rinden cuentas» (Institute on Governance, 2014). En otras palabras, tiene que ver en mayor medida con la forma en que se organiza y estructura la autoridad y la decisión para llevar a cabo ciertos fines definidos por alguien o por algunos (Fukuyama, 2013: 4).

Se trata de una concepción genérica e instrumental acorde incluso con la etimología del término alusiva a manejar o pilotar. En el sentido estricto, la gobernanza es la estructuración de un proceso, sea cual fuere, mediante el cual se toman decisiones. Siguiendo con esta definición como punto de partida, tanto un proceso decisional basado en una sola persona que impone su visión, directa e incluso tiránica, como uno colegiado, discutido y consensuado serían esquemas de gobernanza (Aguilar, 2013: 312). Por ende, se asume que al menos parte de los resultados obtenidos se deberán a la lógica de los mecanismos de decisión que se acordaron o se impusieron, produciendo beneficios o costos para ciertos individuos o grupos.

En su brevedad, la definición citada líneas arriba involucra aspectos en los que la mayoría de los conceptos de gobernanza coinciden. El primero de ellos es que la gobernanza determina quién tiene el poder de tomar o delegar decisiones. El segundo es quiénes intervienen, si hay o no un centro de toma de decisiones y cómo opera dentro de este grupo la rendición de cuentas. Gobernanza refiere, entonces, a cierto mecanismo que define la distribución del poder en un colectivo.

\subsection{Gobernanza democrática o pluricentrista}

Entendido el sustantivo que precede al conjunto de derivaciones del término, resta ahora delimitar los adjetivos que confieren o no ciertos atributos al proceso de toma de decisiones.

En términos simples, el concepto de gobernanza democrática implica un proceso en el que el gobierno, las organizaciones privadas del mercado y la sociedad civil interactúan para decidir, coordinar y llevar a cabo la dirección y gobierno de su comunidad. 
Ante la improductividad e incluso imposibilidad de un gobierno central omnipoderoso, se requiere de una dirección pluricentral o de dirigencia colectiva (Aguilar, 2013: 308).

A diferencia de la gobernanza como mecanismo, el adjetivo democrático integra idealmente a diversos personajes -deseablemente con pesos similares- al proceso de toma de decisiones haciéndolo «mejor», más efectivo y justo. Cómo se encadena esta secuencia (más horizontalidad, mejores resultados) es un supuesto que Fukuyama (2013: 4) advierte debería ser constatado empíricamente y no sólo como deseo. En todo caso, este concepto de gobernanza se refiere en el fondo a mecanismos de participación y equilibrio entre actores diferentes, cuya finalidad es abarcar a todos los implicados en dicho proceso decisional, convirtiendo, aparentemente, a todos o casi todos los gobernados en entes equitativos al menos en su posibilidad de intervención e impacto en el proceso decisional. ${ }^{2}$ En una lógica menos normativa, Aguilar (2013: 310) defiende que el proceso requiere de gobierno pero con una «mayor inclusión de grupos marginados», aceptando que el conflicto aparecerá en dinámicas de gobernanza pluricentrista. Así, es difícil encontrar una argumentación satisfactoria para enlazar causalmente este proceso «ideal» y horizontal con mejores resultados (¿para todos? ¿para la mayoría? son otras preguntas empíricas difíciles de responder hasta este momento). En realidad, la mayor parte de las propuestas de este tipo asumen que un «buen» proceso generará un «buen resultado». Tal vez dicha confianza requeriría ser constatada empíricamente de manera más completa antes de asumir normativamente el resultado.

Llama la atención, siguiendo con este argumento, la sobre confianza que se establece sobre mecanismos de decisión horizontales, participativos y multiactores. Parece, con frecuencia, que dicho proceso de toma de decisiones se convierte prácticamente en un fin: un gran proceso (mayor participación, mayor descentralización, mayor inclusión) que se asume en general «mejor» o «más adecuado» en sí mismo. Bajo esta lógica normativa, los problemas, costos y fallas de la gobernanza democrática o pluricentrista son más difíciles de buscar y de encontrar, pues se corre el peligro de ser atacado como nostálgico del poder central o, peor aún, de antidemocrático.

En la discusión actual existen, también, términos como la buena gobernanza, que se suman al campo de adjetivos que pueden añadirse al proceso de toma de decisiones. Sin embargo, y aunque también se trata de un término aceptado como la gobernanza democrática, obliga de algún modo a preguntarse si, en ese sentido, existe también una mala gobernanza. Si sólo se trata del término simple, una mala gobernanza podría ser aquélla en la cual, sin importar los actores que estén involucrados, realiza también un mal proceso de toma de decisiones.

${ }^{2}$ La Real Academia de la Lengua Española en 2001 se unió también a la tendencia mencionada al cambiar su definición original de «acción y efecto de gobernar o gobernarse» que era más neutral y técnica, a otra normativa: «Arte o manera de gobernar que se propone como objetivo el logro de un desarrollo económico, social e institucional duradero, promoviendo un sano equilibrio entre el Estado, la sociedad civil y el mercado de la economía». Cabría preguntarse qué sociedad y gobierno, en el discurso al menos, no busca exactamente ese desarrollo y ese «sano» equilibrio. Peor aún: como si hubiera un acuerdo académico y evidencias suficientes para decir que puede existir normativamente un "sano equilibrio» como gran solución final a la cuestión gubernamental. Difícil comprender la utilidad de esta definición oficial. 
El término de gobernanza democrática contemporáneo responde no sólo a la pregunta de ¿quiénes toman las decisiones? Sino también a ¿por qué no es únicamente el gobierno el que decide? Esta idea de inevitabilidad de la coordinación entre actores disímiles y contrapuestos en una dinámica «equilibrada» es probablemente el punto fuerte de esta concepción normativa. El dilema justamente consiste en por qué se plantearía por sí mismo como deseable. Habiéndose convertido la democracia en un valor casi incuestionable en Occidente, ¿se asume en automático que la gobernanza democrática es «buena» para todos? ¿Se asume que porque es democrática será y podrá ser justa, equilibrada, pareja (a la Habermas, 1987)? ¿se asume además que al ser democrática será efectiva, eficaz, eficiente para todos los actores involucrados (en contra del principio de la imposibilidad de Arrow (1953), por ejemplo)? Autores como Rothstein (2011) proponen que la única forma de hacer de un proceso un resultado justo es a través del criterio de imparcialidad, justamente porque el dilema clásico de toda democracia no desaparece por una lógica de gobernanza democrática: a saber, la heterogeneidad e irreductibilidad de los valores e intereses de todos los agentes sociales y la dificultad (si no imposibilidad) de crear un mecanismo racional instrumental de agregación de preferencias. En todo caso, una visión más práctica de gobernanza democrática o buena gobernanza, sería la construcción y debate (con evidencias empíricas en mano) de los efectos netos de experiencias de gobernanza de este tipo (Arellano, 2010a). Dicha construcción está todavía por develarse, más allá de la fe en que un mecanismo pluricentrista generará equilibrios e interdependencias «adecuadas».

Respecto de una visión normativa de la gobernanza con una fuerte fe en la lógica de la descentralización o los pluricentros, cabe señalar que ha sido acogida por diversas instituciones y personas con base en muy distintos axiomas y conceptualizaciones. Por ejemplo, el concepto de nueva gobernanza está en realidad más alineado con los supuestos de la nueva gestión pública que posee una visión más precisa respecto del papel de lógicas de mercado, de la regulación y de la no intervención del Estado a menos que sea indispensable (Arellano, 2002). La OCDE (Organización para la Cooperación y el Desarrollo Económico), por ejemplo, plantea la nueva gobernanza como la construcción de inteligentes regulaciones para que actores diversos se comporten de modo tal que cumplan con sus objetivos y, además, realicen tareas y participen con decisiones colectivas, de la mano con el gobierno. Esta lógica puede ser técnicamente resuelta a través de instrumentales como la teoría de redes (gobernanza por redes) y de lógicas participativas (gobernanza participativa y de proximidad). Por último, la Unión Europea, con una visión más pragmática, asume a la gobernanza «como las reglas, procesos y comportamientos que afectan la forma en que el poder es ejercido» pasando con prontitud a asumir que, mientras más abierto y participativo, permitirá que se rinda cuentas de una manera más eficaz (Comisión de las Comunidades Europeas, 2001).

\section{La gobernanza democrática y la lógica de las redes}

El punto de partida o supuesto axiomático básico de la gobernanza democrática o pluricentrista es la incapacidad de los sistemas jerárquicos de gobierno para resolver problemas intrincados que requieren de la participación de muchos actores sociales. En 
el contexto actual, marcado por la complejidad, diversidad y dinamismo, ningún actor -entre ellos, el gobierno- posee los suficientes recursos argumentativos, cognoscitivos, de tiempo, humanos y materiales para imponerse sobre el resto de los actores, mucho menos si lo intenta a través de mecanismos de carácter exclusivamente jerárquicos o formales (Aguilar, 2006). La era de las organizaciones termina y comienza la de las redes (Rosanvallon, 2008:262).

La visión de las redes es una de las más socorridas en este sentido por la visión normativa de la gobernanza (Sørensen y Torfing, 2005): entendida como un conjunto de actores y organizaciones que establecen relaciones de interdependencia de recursos basadas principalmente en mecanismos de cooperación, confianza e inclusive civismo, y no tanto en jerarquías. De ahí que a los actores en una red les resulte natural asociar la interdependencia con la cooperación y coordinación ya que, al estar insertos en un contexto de mayor autonomía (respecto del gobierno) pero con recursos que están dispersos entre una gran cantidad de actores, encontrarán viable y necesario (racional) resolver las posibles diferencias que surjan. Una especie, por tanto, de auto-regulación más que un único diseño central de orden desde arriba.

De algún modo, la perspectiva de la gobernanza democrática supone que los actores - por necesidad, dada la interdependencia- están dispuestos a la participación, es decir, están interesados en un proceso de acción democrática o pluricentral ante la necesidad de coordinarse y vigilarse mutuamente. El gobierno y sus organizaciones son un participante más, tal vez más fuerte y regulador, pero no determinante (Kooiman, 2003). Este hecho tendría consecuencias para la toma de decisiones en el contexto democrático, pues no hay que olvidar que los recursos del gobierno tienen una naturaleza impositiva y están, por tanto, sujetos a diversos controles de índole colectiva (Moore, 1995). Parecería razonable pensar que los actores que compartan la tarea de gobernar también debieran compartir algunos de los controles y tensiones a los que están sujetas las organizaciones gubernamentales. Sin embargo, esto sería aún más complejo, en el entendido de que las redes no pueden considerarse como públicas (Rhodes, 1996). En todo caso, la gobernanza vista desde esta perspectiva requeriría urgentemente mecanismos indirectos de rendición de cuentas a una colectividad más amplia.

En un primer momento y aparentemente no habría problema con la participación de más actores en la toma de decisiones públicas, de acuerdo a diversos autores defensores de esta gobernanza democrática. Las razones son variadas porque la naturaleza de las redes está diseñada para abordar la complejidad de la acción conjunta, por ejemplo. No sólo eso, también se parte del supuesto de que garantizaría una mayor y mejor información (Rhodes, 1996), además de un mejor uso de los recursos. La lógica sería llegar a acuerdos sobre los valores y objetivos principalmente a través de negociaciones, apoyadas por fuertes lazos de confianza (Rhodes, 1996). El mejor uso de la información y demás recursos (Kooiman, 2003) favorecerían la transparencia, los temas ganarían más visibilidad para vincularse con un colectivo más amplio, aunque se reconoce que la información podría estar condicionada. Así, dos de los prerrequisitos para la acción democrática estarían colocados.

Una vez que la participación está asegurada -no necesariamente con simetría de participación, pues las diferencias de recursos son un producto sedimentado de una 
larga historia social (Aguilar, 2006)- los mecanismos de la gobernanza permitirían un abordaje más comprensivo de las diferencias entre los participantes en el sentido de que se podría sensibilizar a los actores con más recursos para convertirse en unos más receptivos hacia los participantes menos favorecidos, hecho que tendría efectos positivos en la igualdad y en la equidad (O'Toole, 1997). El intercambio de recursos y de información redundaría en que las organizaciones y los actores contarían con mayores elementos y se reducirían las asimetrías de información: habría una permanente comunicación de ida y vuelta entre los actores (Hirst, 2000, citado en Aguilar, 2006). Esta interdependencia crearía lazos que reforzarían la responsabilidad conjunta, de modo que los actores pudieran facilitar la fase de implementación y no obstaculizarla ((Sørensen \& Torfing, 2003, 614, citado en Sørensen y Torfing, 2005). Se cuenta, desde este enfoque, con incentivos idóneos para que los actores reconozcan que el problema tiene significación social (Aguilar, 2006).

$\mathrm{Al}$ asumir las redes mayor relevancia en la provisión de diversos bienes y servicios, habría espacio para que las organizaciones gubernamentales concentraran sus esfuerzos en tareas de supervisión y facilitamiento. El mejor aprovechamiento de recursos junto con el involucramiento de los actores relevantes por parte de instituciones del mercado y la sociedad civil garantizaría una mayor eficacia y calidad de los servicios, redundando así en una fuente de legitimidad en términos democráticos. En forma implícita, se reconoce en el gobierno un rol de vigilante del sistema de redes con la capacidad de influir y disponer de incentivos, así como de un sistema de reglas que propicien una mayor eficiencia y eficacia, incluso en aquellas sociedades donde éstas, las redes, no han surgido lo suficiente, bien sea por cuestiones históricas o culturales. Los incentivos para lograr la cooperación y la articulación de la red de organizaciones existen, es cuestión de encontrarlos.

La capacidad de adaptación de las redes sería un elemento adicional que fortalecería la acción democrática (Kooiman, 2003). En general, se atribuye a las redes una gran capacidad de flexibilización que les permitiría adaptarse fácilmente ante las nuevas disposiciones que surjan en el contexto democrático, o incluso prever problemas y descubrir oportunidades en determinados momentos. Su naturaleza abierta las orientaría - en menor medida - a la entropía, en el entendido de que, al ser parte de un proceso que incluye diálogo mutuo y constante, la acción democrática no sería algo que ocurre sólo en el periodo de elecciones, sino de manera permanente (Peters, 1996). En el proceso mismo se descubrirían mecanismos de metagobernanza que asegurarían en todo momento que las interacciones sociales se encaminasen hacia objetivos y metas compartidas. Dicho en otras palabras: en todo momento se tendría no sólo conciencia de una situación concreta y las posibles diferencias con la situación deseada, sino también de los instrumentos para lograr la convergencia entre el diseño y la realidad concreta.

En suma, la gobernanza y la acción democrática serían planteadas a partir de diversos supuestos basados en el funcionamiento de las redes. Se asume que existen (o se pueden instaurar al menos) los incentivos y las reglas, incluso de tipo moral, para facilitar y dar coherencia a la acción democrática a partir de la gobernanza, en un proceso -matizado por Prats (2005)- de «experimentación y aprendizaje por prueba y error». Si pudiera resumirse la dinámica de la gobernanza y su relación con la acción 
democrática sería a través de los beneficios comúnmente asociados con las redes: apertura, participación, responsabilidad, eficacia y coherencia (Comisión de las comunidades europeas, 2001).

Visto como una red o conjunto de redes, la horizontalidad o pluricentralidad de la gobernanza requiere siempre una comprensión más integral del papel del poder y el peso de los actores involucrados en un contexto particular (Wachhaus, 2013). Para comenzar, gobernanza no es en realidad un concepto contrapuesto al de gobierno (Offe, 2009: 551). Olsen (2006) habla, por ejemplo, de una reexaminación y redefinición de las opciones que forman parte de un nuevo equilibrio institucional, un reposicionamiento de los equilibrios como parte de movimientos pendulares de las batallas por el poder y por el control social del poder.

Comenzando por el papel del gobierno, no como un monolito sino como una red flojamente acoplada de organizaciones, es preciso añadir que dichas organizaciones son criaturas políticas obligadas, como toda organización, a adaptarse a su contexto y generar viabilidad y valor público en forma estratégica (Moore, 1994). La naturaleza política de las organizaciones gubernamentales es una variable crítica para comprender en la práctica la interacción en red de diversos actores sociales. No siendo el gobierno un monolito, las organizaciones públicas enfrentan decisiones y riesgos, éxitos y fracasos (Olsen, 2006). La burocracia vista en acción es mucho más que jerarquía, rigidez y decisiones unilaterales (Du Gay, 2012).

\section{Gobernanza: el factor organizacional como tránsito necesario}

Cuando algunos textos contemporáneos sobre gobernanza asumen, como si fuera algo nuevo (supuesto cuestionable), que las organizaciones de la sociedad civil y las del mercado compartan la tarea de generar acciones y logros de interés colectivo, surgen diversas implicaciones que deben ser consideradas en un contexto incierto de dinamismo, complejidad y diversidad como el presente. La pregunta principal es si las características comunes a las organizaciones más las especificidades de la naturaleza pública, propias de ellas, hacen viable que la propuesta de la gobernanza promueva sistemáticamente la acción democrática. En otras palabras, incorporar la variable organizacional implica abandonar -al menos por un momento- una visión instrumentalista y voluntarista del diseño racional de instituciones. Si bien cualquier acción organizada actúa en el hoy y en las circunstancias presentes, los efectos de su acción son afectados por las interactuaciones con otros actores y con diversos contextos, generando ineludiblemente efectos no esperados y no buscados. Introducir la variable organizacional es una de las maneras clásicas en los estudios de administración pública para comprender cómo todo proyecto, por más racionalista que sea, termina produciendo

${ }^{3}$ En realidad, la variable organizacional va más allá del gobierno como red flojamente acoplada de organizaciones. Las organizaciones sociales y privadas que participan en una red de gobernanza requerirían también ser comprendidas en una lógica similar. Sin embargo, dicha tarea está más allá de las posibilidades del presente artículo. 
efectos y resultados distintos a los que se esperaba (Pressman y Wildavsky, 1984). Una perspectiva de gobernanza requiere, entonces, enfrentar estos elementos que, de manera condensada, denominaremos como «el tránsito» organizacional. Se trata de estudiar los retos que una perspectiva de gobernanza democrática tendría que resolver, desde el ángulo organizacional, para pasar de una lógica discursiva y normativa a una más empírica y aplicada, capaz de enfrentar la contingencia ${ }^{4}$ propia de los fenómenos sociales. Analicemos tres zonas de tránsito de la dinámica organizacional ${ }^{5}$.

\subsection{La incrustación a la dinámica organizacional}

La vida social contemporánea no podría entenderse sin las organizaciones. Ellas no son sólo instrumentos para producir bienes y servicios. Son, además, mecanismos a través de los cuales las sociedades generan sentido a un cúmulo de interacciones sociales que buscan conseguir ciertos resultados o productos (Weick, 2001). Estas interactuaciones se generan entre agentes sociales heterogéneos y diversos, no transparentes ni motivados por un solo deseo o interés. El acto social y organizacional es uno de naturaleza política y opaca, en ocasiones estratégica. Es un mundo de representaciones e interpretaciones, de máscaras y engaños necesarios y estratégicamente construidos (Goffman, 1981). El mundo de la micro acción, de los micro-comportamientos es probablemente uno de los aspectos más olvidados o subestimados por disciplinas como la administración pública o la ciencia política contemporánea. Aspectos que son críticos también para comprender la dinámica concreta de cuestiones como la gobernanza democrática.

La sección que precede a ésta explicó que la gobernanza democrática concede a las redes una amplia capacidad de inclusión. Con ello, favorecen de facto el principio democrático de mayor equidad de participación. No hay que olvidar, sin embargo, que las redes, al estar compuestas por organizaciones y actores, difícilmente escaparán a la dinámica política y de poder que amalgama la acción organizacional (Crozier y Friedberg, 1980). La viabilidad de la interacción en las organizaciones se genera en un proceso de reconstrucción política de espacios de poder e incertidumbre: los actores organizacionales ganan poder al controlar arenas de incertidumbre en juegos políticos de interdependencia.

Uno de los aspectos más importantes que propone la gobernanza es la toma de decisiones compartida por el gobierno, organizaciones civiles y el mercado. Sin embargo, esta integración de los dos últimos grupos de agentes mencionados es formulada como un paso automático y sin sobresaltos en términos teóricos. En el terreno empírico, compartir la tarea de gobernar o tomar decisiones implica integrar organizaciones

${ }^{4}$ Véase el análisis crítico que hace Aguilar (1981: 27-34) sobre el debate en la filosofía clásica alemana sobre el deber ser y la realidad contingente.

5 Aguilar, en su excelente artículo (2014), aborda la importancia de la gobernanza del aparato administrativo como un todo y como un grupo de organizaciones. En este artículo nuestro, sin embargo, preferimos introducir la literatura organizacional que habla de la acción más que del deber ser gerencial. 
-civiles o privadas- a otras organizaciones - gubernamentales- de modo que se construya una metaorganización: la gobernanza. Esta dinámica se genera, en la arena empírica, a través de un proceso de interdependencia similar al que se ocurre en una organización para detonar la acción cooperativa. Pero dicho proceso en una organización es un proceso político de lucha y disenso. ¿Qué lo haría distinto en la metaorganización de gobernanza?

La interacción y creación de interdependencias propios de la idea de gobernanza democrática requiere analizar y teorizar sobre el conflicto propio de un proceso de interrelación de agentes distintos y heterogéneos. Crear un acuerdo entre actores que tienen diferencias entre sí es posible, pero no es un proceso automático y tampoco culmina con el acuerdo de las decisiones y su estructuración: se genera también en la acción, en la evaluación de resultados y efectos concretos de la interdependencia. Estructurar la decisión es un paso importante, sin duda, pero no suficiente: la estructuración de la acción es un paso relacionado pero distinto que requiere de otras habilidades, acuerdos y de nuevos conflictos (Brunsson, 2007a).

El paso de decisión a acción es, en realidad, menos lineal de lo que se asume regularmente. Hay decisiones sin acción, acción sin decisiones y efectos de acciones que no se relacionan con decisiones. Las decisiones son una institución, dice Brunsson (2007a), y con ello señala que las organizaciones han construido un mundo simbólico en el que la decisión dirige y precede a la acción, cuando en la práctica la relación entre ambas es compleja y no directa ni racional necesariamente. En algunas ocasiones incluso, la construcción de acciones viables requiere de simulación, muchas veces de hipocresía, exageración, manipulación de emociones e incluso mentiras piadosas de engaño (Goffman, 1981). La acción es un claroscuro donde diversos agentes entran en relaciones de muy diversos tipos, enfrentando situaciones, contingencias y multiplicidad de intenciones. Haya sido el proceso de decisión horizontal, democrático o pluricentrista, el tránsito organizacional se habla en el lenguaje del poder, el conflicto y la lucha formal e informal por el control de la incertidumbre incluso a través de mentiras, engaños, simulaciones y máscaras.

El secreto de la acción radica, entonces, en la interacción, en su situacionalidad y contingencia. La interacción necesita que los agentes se agrupen, busquen alianzas, construyan muros, tejan compromisos y valores. En este sentido, la inclusión exitosa a la organización, en términos generales, implica una diferenciación o una toma de partido, entendida como la adhesión a ciertos grupos, donde el elemento diferenciador entre un grupo y otro es, además de un conjunto determinado de valores, el secreto o la información que uno guarda (o inclusive que aparente guardar) frente al otro. Curiosamente, el proceso de inclusión en lo general produce escenarios de diferenciación y exclusión en lo particular y hacia dentro de las organizaciones. Es ahí donde los actores, los grupos y las organizaciones tratarán de esconder los errores, lo desordenado de los procesos, los sacrificios de valores y las falsas expectativas (Goffman, 1981).

La gobernanza pareciera suponer que los actores serán del todo transparentes desconociendo la función del secreto para ayudar a configurar las interacciones. Contrario a este supuesto es pertinente apuntar que, llevado al extremo, el secreto puede redundar en acciones de consecuencias imprevisibles para la acción democrática. 
El proceso de incrustación y permanencia en la dinámica organización depende, en estos términos, de la capacidad de los actores para internalizar roles que tengan que desempeñar, asumiendo que el secreto y la representación son elementos indispensables en la interacción (con base en Arellano, 2010). Una dramatización a destiempo o compartir demasiada información puede, en cualquier momento, acabar con la interacción: una sola nota desatinada puede destruir el tono de toda la actuación (Goffman, 1981). Los actores y organizaciones mejor preparados para desempeñar los roles serán aquéllos capaces de crear o inducir situaciones a partir de la capacidad de interactuar e interpretar un contexto que no es del todo lineal ni transparente: de asegurar de algún modo la reciprocidad (Arellano, 2010). Es por ello que algunos críticos de la gobernanza no dejan de señalar que siempre existirán actores capaces de manipular gracias a sus capacidades o posiciones especiales en la arena social (por ejemplo, los expertos, según explica Shapiro, 2001).

La apuesta de la gobernanza al hecho de que las organizaciones gubernamentales deben incetivar la participación -más aún, generar un entorno de sistema de redes a partir de incentivos- contrasta con la dinámica de incrustación de las organizaciones donde el secreto y la representación requieren ciertas habilidades especiales con las que no todos los actores cuentan. Olivo (et. al., 2011) expone cómo una política inspirada en la gobernanza que da por hecho la participación sin considerar las expectativas de los actores puede terminar en mera simulación y en un gasto innecesario de las energías sociales. Wachhaus (2013) defiende que, si bien contar con una perspectiva que incluya amplia participación -desde una postura anárquica, inclusive- puede mejorar el proceso de acción del gobierno, dicha participación debe ser un ejercicio voluntario y consciente de los actores.

En un resumen de trazos gruesos, la búsqueda de incluir a todos o a la mayoría de los actores en el discurso de la gobernanza democrática requeriría pasar de la esperanza normativa a la práctica de la interacción y, por tanto, de la dinámica organizacional.

\subsection{La toma de decisiones y la acción organizacionales}

La acción organizacional es un proceso complejo de relaciones, actores, actuaciones, intereses, estrategias, disímbolas y heterogéneas. En un gran número de ocasiones, tomar decisiones y pasar a la acción precisa negociaciones y cooptaciones (Selznick, 1949).

Siendo las organizaciones espacios de cooperación pero también -y principalmente- de competencia por los fines y los medios, el proceso de decisión y de acción implica amplios espacios para la irracionalidad, la discrecionalidad y la opacidad. Aspectos de la situación y estrategias de los actores, al mismo tiempo, para enfrentar una realidad más dinámica y desordenada de lo que asumen perspectivas normativas como la gobernanza.

Brunsson (1986) advierte que, en las organizaciones, no siempre se toman decisiones en forma racional. Es decir, que los actores no cuentan completamente con un marco de definiciones (en términos de lo correcto y lo incorrecto) operativas incontrovertibles de los objetivos, de las metas y los efectos de las acciones y decisiones pre- 
sentes y futuras (Arellano, 2010:76). Tampoco cuentan con el involucramiento constante y previsible de otros actores (Cohen, March y Olsen, 1972). En un escenario como éste, no es de extrañarse que la toma de decisiones y la acción difieran del ideal. En la mayoría de los casos, los objetivos permanecen difusos o se descubren a través de la acción misma (Weick, 2001 citado en Arellano, 2010a), e incluso si se tuviesen algunas definiciones de los fines y los medios, éstos últimos necesariamente serán interpretables, dado que los actores cuentan con racionalidad limitada. Aunado a ello, los actores entran y salen dependiendo, en buena medida, de la disponibilidad de tiempo y la capacidad de esfuerzo, por lo que los límites de las organizaciones son siempre inciertos, móviles y confusos (Cohen, March y Olsen, 1972).

Sin embargo, no todo es irracionalidad en las organizaciones. Brunsson (1986) explica cómo la perspectiva racional es muy útil para los actores al momento de reflejar la pluralidad de los intereses en las organizaciones pero es, de manera simultánea, de poca utilidad para generar el consenso que requiere la toma de decisiones y la acción conjunta. Lo racional, entonces, permite exponer la complejidad que implica tratar con actores con distintas creencias, intereses y expectativas. El problema, visto de este modo, es que la racionalidad tiende a abrumar a los actores y, eventualmente, los conduce a la parálisis en la toma de decisiones y la acción (Brunsson, 1986). El consenso implica motivación, entusiasmo, responsabilidad y compromiso. Estas cualidades para la toma de decisiones y la acción necesitarían cierta irracionalidad (Brunsson, 1985), pero esta irracionalidad podría, a su vez, asociarse con problemas de mala calidad de la decisión (Moore, 1995).

Finalmente, la irracionalidad es potenciada por un marco de relaciones informales que necesariamente sobrevienen cuando los actores están expuestos a la interacción constante, especialmente para sobrellevar un contexto marcado por la pluralidad de intereses. Los problemas públicos llevan consigo una multidimensionalidad de factores, lo que conduce a las organizaciones a una acción discrecional y a la flexibilidad. El primer elemento, la irracionalidad, aunado a la red de relaciones informales comentada, propicia la aparición del segundo elemento: la discrecionalidad.

\subsection{Discrecionalidad}

La informalidad es indispensable para fortalecer la comunicación (Barnard, 1938) y para favorecer la reciprocidad en el tiempo, además de ser uno de los principales mecanismos que tejen los actores para entender y encontrar sentido a la propia acción organizada (Weick, 2001). El problema de la informalidad es que, sin contrapesos claros, las organizaciones buscarán sustituir el trato impersonal por las necesidades personales, campo ideal para la fenómenos que van desde la corrupción y el egoísmo (con base en Clegg et al., 2006; en Arellano, 2010) hasta el cinismo o la hipocresía (Moore, 1995), el nepotismo, favoritismo (Merton, 1999) o la cooptación (Selznick, 1949), todos ellos pretextos para actuar fuera de las reglas, con claras implicaciones legales y para la propia acción democrática (Moore, 1995).

El riesgo de favorecer a una minoría bien organizada antes que a grupos o actores aislados es por demás evidente. Aceptar la informalidad en las organizaciones por las 
ventajas que ésta ofrece implica, de manera simultánea, aceptar la posibilidad de discrecionalidad e irracionalidad en la toma de decisiones y la acción en las organizaciones. El énfasis de la gobernanza en la informalidad como forma que facilita la interacción entre los actores comúnmente olvida el lado oscuro de ésta misma, así como las consecuencias que tiene para la toma de decisiones y la acción organizacionales.

\subsection{Información}

La información, su producción y diseminación constituye un tercer elemento propio de las organizaciones que contrasta con el discurso de la gobernanza. Los intereses y preferencias de los actores no son estáticos ni consistentes. Pueden, además, ser ambiguos o estar mal definidos (Dahl, 1947) y, con frecuencia, se encuentran en constante reevaluación en función de los flujos de información y de las interpretaciones que hacen de la situación (Cohen, March y Olsen, 1972). En un contexto plural y dinámico con las características mencionadas, la información se convierte en uno de los principales valores estratégicos (Crozier, 1974). Así, los esfuerzos por contar con mayores elementos que permitan reducir los espacios de incertidumbre sobre las consecuencias de las decisiones -siempre impredecibles (March y Olsen, 1984)-, lleva a unos actores a buscar información en posesión de otros actores. Al tratarse de un proceso que demanda tiempo y otros recursos, estos personajes entienden el valor estratégico de la información y, dada su estima, someterán su disposición a compartirla o denegarla en las negociaciones (Crozier y Friedberg, 1980). En otras palabras, la información forma parte de un juego de ofensivas y defensivas que los agentes organizacionales lanzan con el fin de mejorar sus posiciones a costa de otros o en coaliciones con otros.

\subsection{La irracionalidad, discrecionalidad e información como elementos necesarios en la toma de decisiones y acción organizacional}

En contextos de pluralidad -ya existentes incluso antes de añadir los principios planteados por la gobernanza-, las organizaciones no pueden escapar de dos lógicas opuestas: una que implica la búsqueda de consenso para la acción conjunta, mientras que otra resuelve las diferencias que surgen cuando se confrontan distintos intereses y preferencias entre los actores organizacionales (Brunsson, 1986). El conflicto se convierte, entonces, en un elemento endémico. Los acuerdos son pasajeros, tenues (Cyert y March, 1992). La toma de decisiones redunda en poca o escasa sincronía con la acción organizacional o con los actores que la desencadenan. De ahí que algunas decisiones surjan al vuelo o por descuido (Cohen, March y Olsen, 1972), después de la acción misma, interpretando (Weick, 2001). Naturalmente, cualquier mecanismo de control y coordinación establecido previamente corre el riesgo de colapsar ante esta dinámica (Cohen, March y Olsen, 1972). El hecho de que las organizaciones sean espacios donde buena parte de las decisiones son producto de acontecimientos imprevistos, senderos tortuosos, luchas intestinas, múltiples participantes y consideraciones extravagantes (Mény y Thoenig, 1992:131) las convierte en atenuadores o disipadores 
de responsabilidad (O’Toole, 1997) o de incertidumbre jurídica, incluso, pues es difícil vincular una decisión o acción con los responsables. Visto de este modo, la irracionalidad que es criticada en la toma de decisiones al interior de las organizaciones es un mal necesario para evitar la parálisis y buscar el consenso.

Ahora bien, desvincular la toma de decisiones de la acción puede redundar en distintos escenarios. Uno de ellos es aquél en el que las decisiones no siempre se tomen en función de un consenso, sino por imposición de grupos y actores que aprovechan la capacidad de controlar los espacios de incertidumbre a su alcance (Crozier y Friedberg, 1980). Un escenario más es aquél en el que los fines y los medios puedan ser atrapados por grupos o actores con mayores recursos: la capacidad de veto en los puntos de decisión por parte de grupos con recursos estratégicos está siempre presente (Pressman y Wildavsky, 1984).

La separación de estos mecanismos redunda en una segregación de la participación y el contenido de los resultados de la interacción organizacional que aplica a los actores, necesariamente interesados en facetas de determinados procesos y no en la totalidad. Algunas acciones estarán dirigidas a grupos específicos, mientras que otros grupos habrán de conformarse sólo con una parte de la toma de decisiones: los discursos. Esto no significa, por supuesto, que los tres elementos -discurso, toma de decisiones y acción- sean coherentes entre sí. Pueden llegar incluso a ser contradictorios, y habrá en ellos espacio para la simulación, la hipocresía y la justificación (Brunsson, 1986) de manera que puedan acoplarse conforme a la necesidad del instante.

Lo anterior podría desconcertar a un observador externo que pensará que los vínculos entre discurso, toma de decisiones y la acción debieran seguir un patrón racional. Sin embargo, las organizaciones están inmersas en un proceso que va más allá de los resultados, de la acción, y que incluye al proceso político mismo como uno de sus objetivos finales (Olsen, 2005). De ahí que las organizaciones, al recabar información y trazar planes, actúan más en el sentido de la lógica de lo apropiado que en la búsqueda de resultados o en la satisfacción del proceso mismo de toma de decisiones (Feldman y March, 1981, citado en March y Olsen, 1984).

La irracionalidad no es, entonces, una patología sino una respuesta que muestra claramente cómo - en cualquier contexto de pluralidad y dinamismo- cualquier intento de mecanismo racional de toma de decisiones y acción organizacional tiende a colapsar casi irremediablemente (Cohen, March y Olsen, 1972). Es en este contexto donde opera el control y coordinación organizacionales. La supuesta capacidad de la gobernanza para obtener resultados, ser eficaz y resolver problemas tiene necesariamente que enfrentarse a esta dinámica de la toma de decisiones y la acción organizacional: la dificil tarea de optimizar el funcionamiento de las redes de gobernanza en todas sus dimensiones (Klijn y Koppenjan, 2004:114 citado en Sørensen y Torfing, 2005).

Es posible prever que prevalecerán aquellos actores con mayores capacidades de recursos, tanto cognoscitivos como informativos, retóricos y materiales: aquéllos que logren controlar espacios de incertidumbre a través de cierto dominio sobre los recursos (Olson, 1992). Los arreglos legales y demás mecanismos formales pueden ser rígidos, pero están pensados para limitar precisamente estas acciones de poder (Crozier y Friedberg, 1980). La rigidez característica de las organizaciones, criticada en lo nor- 
mativo por la gobernanza, termina siendo una defensa en lo empírico de uno de los valores que la misma gobernanza contempla, la inclusión equitativa. Agregar a esta lógica simplemente más actores con la esperanza de que su simple horizontalidad o sus conexiones en redes resolverán esta lógica de la naturaleza organizacional puede ser considerado relativamente ingenuo. En todo caso, dicha resolución de la dinámica intrincada y conflictiva de las organizaciones a partir de lógicas de gobernanza debería construirse y defenderse, más que asumirse o subsumirse.

\section{Conclusiones}

Es difícil estar en desacuerdo con la esperanza de que un enfoque de gobernanza horizontal sea una pieza para mejorar la capacidad y calidad del gobierno -en conjunto con la sociedad civil y las instituciones de mercado- para el tratamiento y eventual solución de problemas colectivos públicos. Sin embargo, una vez que se revisan los fundamentos teóricos, los supuestos, los mecanismos, las prioridades y las responsabilidades, el escepticismo emerge prontamente.

En este artículo hemos dibujado una serie de dudas respecto de la viabilidad práctica de una visión normativa de la gobernanza, como si de alguna manera la lógica política de la interacción humana se viera controlada y neutralizada por una idea de horizontalidad en las decisiones y en las acciones colectivas. En concreto, hemos apuntado hacia la duda razonable de que la esperanza horizontalista de decisiones en realidad afecte de manera sustantiva el mundo intrincadamente político y social de las decisiones y las interacciones humanas en una sociedad plural. Hemos puesto para ello a las organizaciones al frente, cuestionando la idea de que las redes sustituyan a las organizaciones: las organizaciones siguen siendo estas relaciones sociales tremendamente efectivas para dar sentido a la acción en un mundo plural y bajo constante incertidumbre.

Nuestra perspectiva argumenta, entonces, que más que hablar de la gobernanza deberíamos hablar de varios tipos de gobernanza, todos como instrumentos con pros y contras y profundamente situacionales. Es decir, afectados por los detalles de la implementación, el contexto, la personas y las vicisitudes de la acción propiamente dicha. En otras palabras, plantear únicamente sus beneficios en la arena de lo teórico es insuficiente: mecanismos de gobernanza que en el papel suenan altamente participativos, horizontales y efectivos, en la acción se pueden convertir en pesadillas de implementación o generadores de efectos netos negativos.

La propuesta consiste en ir más allá del debate de las virtudes de un discurso únicamente. Habría que tomar una posición de búsqueda de evidencia empírica (Graña, 2005), preparados para encontrar efectos positivos pero probablemente también negativos. Quizá sea adecuado, desde la discusión normativa y pensando siempre en su progresión empírica, pasar de la básica discusión de «gobernanza, ¿sí o no?» hacia una del tipo: «¿qué tipos de gobernanza para qué situaciones?»; y todavía más importante, «cada tipo de gobernanza, ¿cómo?» La propuesta de los estudios de gubernamentalidad por ejemplo, puede ser una manera prometedora de abordar esta discusión: estudiar la gobernanza no sólo como un grupo de instituciones y un proceso normativo sino una actividad de poder práctica a través de programas racionales y técnicas de domi- 
nación y disciplina (Walters, 2012:2). En este caso (seguramente hay otros) la idea de gubernamentalidad tiene la ventaja de enfatizar la importancia de la política más allá de una visión paradójica de la gobernanza como un proceso político pero anti-político: como si la horizontalidad acabara o diluyera la dominación.

Ahora bien, nos queda volver a las organizaciones. El análisis teórico de la dinámica organizacional acompañado de algunas experiencias revela diversos elementos de riesgo porque éstas exhiben una lógica intrincada, no racionalista ni sencilla de dirigir o diseñar. Y eso hace difícil para este enfoque de la gobernanza resistir inicialmente al menos la prueba del tránsito organizacional. Las organizaciones tienden a ser espacios de negociación, poder, irracionalidad, discrecionalidad. Estos son ingredientes fundamentales para el logro de los objetivos organizacionales, no patologías. De modo que los actores y las organizaciones no son instrumentos técnicos que reaccionan sin más a los incentivos y al diseño institucional, por más elaborado que éste pueda ser.

Si estas dudas que hemos planteado tienen sentido, vale la pena retornar al debate de la gobernanza con ojos menos normativos y haciéndose preguntas hacia adelante. Por ejemplo: ¿estas ideas de gobernanza democrática sufrirán, como toda propuesta social, consecuencias no esperadas y no deseadas de la acción? Nuestra apuesta es que sí, y por tanto, valdrá la pena llamar la atención desde ahora y discutirlo desde el principio. Una pregunta más podría enunciarse como ¿puede un concepto de gobernanza de este tipo abonar el descrédito de la acción gubernamental y la frustración de los ciudadanos al proponer una visión ingenua, irrealizable e impráctica del acuerdo en sociedades plurales? (lo que Rosanvallon, 2008: 264 llama legitimación destructiva.) Pareciera más sensato plantearse a la gobernanza menos como una gran solución alternativa ideal para todo tipo de realidades políticas y más como un instrumento, un esquema alternativo, con ventajas y desventajas incorporadas.

\section{Bibliografía}

Acemoglu, D y Robinson, J. (2012). Why nations fail: the origins of power, prosperity and poverty. Crown Publishers, Nueva York.

Aguilar Villanueva, L. F. (1981). Weber: la idea de ciencia social. Vol 1. Miguel Ángel Porrúa, México.

Aguilar Villanueva, L. F. (2006). Gobernanza y gestión pública. FCE, México.

Aguilar Villanueva, L. F. (2013). Gobierno y administración pública. FCE-CONACULTA, México.

Aguilar Villanueva, L. F. (2014). «Las dimensiones y los niveles de la gobernanza», en Cuadernos de Gobierno y Administración Pública, volumen 1, número 1, pp. 11-36.

Arellano, D. (2002). «Nueva Gestión Pública: ¿el meteorito que mató al dinosaurio? Lecciones para la reforma administrativa en países como México», en Reforma y Democracia, $\mathrm{n}^{\circ} 23$, pp. 7-40.

Arellano, D. (2010). «El enfoque organizacional en la política y gestión públicas. Entendiendo las organizaciones gubernamentales», en Merino, M., y Cejudo, G., (compiladores.), Problemas, decisiones y soluciones. Enfoques de política pública, FCE, México.

Arellano, D. (2010a). «Reformas administrativas y cambio organizacional: hacia el «efecto neto», en Revista Mexicana de Sociología, no 72, vol. 2, pp. 226-254. 
Arrow, K. (1953). Social choice and individual values. Yale University Press, New Haven.

Banco Mundial. (1997). World Development Report: The State in a Changing World. Disponible en línea en http://wdronline.worldbank.org//worldbank. Nueva York

Barnard, C. (1938). «Las organizaciones informales y sus relaciones con las organizaciones formales», en Shafritz, J.M. y Hyde, A., eds. Clásicos de la administración pública, traducción María Antonia Neira Bigorra. Colegio Nacional de Ciencias Políticas y Administración Pública, FCE, México.

Bowen, R. (ed.) (1971). German Theories of the Corporative State. Russell \& Russell, Nueva York.

Brunsson, N. (1985). The irrational organization. John Wiley and Sons, Chichester.

Brunsson, N. (1986). "Organizing for inconsistencies. On organizational conflict, depression and hypocrisy as substitutes for action" en Scandinavian Journal of Management Studies, $\mathrm{n}^{\mathrm{o}} 2$, vol. $3 / 4$, pp. 165-185.

Brunsson, N y Olsen, J. (2007). La reforma de las organizaciones. CIDE, México.

Brunsson, N. (2007). Consequences of decision making. Cambridge University Press, Cambridge.

Brunsson, N. (2009). Reform as Routine. Organizational change and stability in the modern world, Oxford University Press, Oxford.

Canto Sáenz, R. (2012). "Gobernanza y democracia: De vuelta al río turbio de la política" en Gestión y política pública, México, n 21, vol. 2, pp. 333-374.

Clegg, S. et al. (2006). Power and organizations. Sage, Londres.

Christensen, Ty Laegreid, P. (2007). Organization theory and the public sector. Rutledge, Londres.

Cohen, M, March, J y Olsen, J. (1972) “A garbage can model of organizational choice", en Administrative Science Quarterly, $\mathrm{n}^{\circ}$ 17, vol.1, pp. 1-25.

Comisión de las comunidades europeas. (2001). La gobernanza europea, un libro blanco, Bruselas.

Crozier, M. (1974). El fenómeno burocrático: ensayo sobre las tendencias burocráticas de los sistemas de organización modernos y sus relaciones con el sistema social y cultural. Amorrortu, Buenos Aires.

Crozier, M y Friedberg, E. (1980). Actors and systems: the politics of collective action. University of Chicago Press, Chicago.

Cyert, R y March, J. (1992). A behavioral theory of the firm. Blackwell, Nueva York.

Dahl, R. (1947). «The Science of public administration», en Public Administration Review, ${ }^{\circ}$ 1, vol. 7.

De Alcántara, C. (1998). "Du bon usage du concept de gouvernance”, en Revue Internationale de Sciences Politiques, $\mathrm{n}^{\circ} 155$, pp. 109-118.

DiMaggio, P y Powell, W. (1983). The new institutionalism in organizational analysis. Chicago University Press, Chicago.

Du Gay, P. (2012). Elogio de la burocracia. Siglo XXI, Tres Cantos, Madrid.

Elster, J (2007). Explaining social behavior. More Nuts and Bolts for the Social Sciences. Cambridge University Press, Cambridge.

Fukuyama, F. (2013). What is governance? Working paper 314, CGD, Washington DC.

Fukuyama, F. (1992). The end of history and the last man. Free Press, Nueva York.

Feldman, M y March, J. (1981). «Information as signal and symbol», en Administrative Science Quarterly, n 26, pp. 171-186.

Flyvbjerg, B. (1991). Rationality and power. Democracy in practice. Chicago University press, Chicago. 
Garland, R. (2009). Project Governance - a practical guide to effective project decision making-. Kogan Page, Londres.

Goetz, K. (2008). "Governance as Path to Government, en West European Politics, vol. 31, n” 1, vol. 2, pp. 258-79.

Goffman, E. (1981). La presentación de la persona en la vida cotidiana. Amorrortu, Buenos Aires.

Goodin, R. (1996). The theory of institutional design. Cambridge University Press, Nueva York.

Graña, F. (2005). "Todos contra el Estado: Usos y abusos de la «gobernanza»", en Espacio Abierto, Octubre-Diciembre, pp. 501-529.

Grindle, M. (2010). Good governance: the inflation of an idea. CID. Working Paper no 202. Harvard University, Cambridge.

Gulick. L. (1937). "Notas sobre la teoría de la organización”, en Clásicos de la administración pública, Colegio Nacional de Ciencias Políticas y Administración Pública A.C.- FCE, pp. 189-205, México.

Habermas, J. (1987). Teoría de la acción comunicativa. Taurus, Madrid.

Hanford, M. (2006) Establishing portfolio management governance: Key components. Developer works. Disponible en ibm.com/developerworks/rational/library/oct06/hanford/ index.htm IBM. Estados Unidos.

Hirst, P. (2000). Associative Democracy: New Forms of Economic and Social Governance. University of Massachusetts. Amherst.

Hood, C. (1991). “A Public Management for All Seasons?” en Public Administration, n 69, vol. 1, pp. 3-19.

Hood, C. (2011). The blame game: spin, bureaucracy, and self-preservation in government. Princeton University Press. Princeton

Institute on Governance. (2014). Defining governance. Disponible en http://iog.ca/aboutus/defining-governance/ Canadá.

Kagancigil, A. (1998). "Gouvernance et science: modes de gestion de la société et de production du savoir empruntés au marché", en Revue Internationale de Sciences Politiques n ${ }^{\circ}$ 155. UNESCO, pp. 73-84.

Klijn, E. H y Kopperjan, J. (2004). Managing Uncertainties in Networks. Routledge, Londres.

Koch, P. (2013). "Overestimating the Shift from Government to Governance: Evidence from Swiss Metropolitan Areas", en Governance, no 26, vol. 3, pp. 397-423.

Kooiman, J. (2003). "Governing as Governance", ponencia presentada en la Conferencia Internacional Gobernanza, Democracia y Bienestar Social Barcelona: Institut Internacional de Governabilitat de Catalunya.

March, J y Simon, H. (1993). Organizations, Blackwell, Cambridge.

March, J y Olsen, J. (1976). Ambiguity and Choice in Organizations. Universitetsforlaget, Bergen.

March, J y Olsen, J. (1984). "The New Institutionalism: Organizational Factors in Political Life", en American Political Science Review, n 78, vol. 3, pp. 734-749.

March, J y Olsen, J. (1989). Rediscovering Institutions. Free Press, New York.

March, J y Olsen, J. (2006). "The Logic of Appropriateness", en Michael Moran, Martin Rein, and Robert E. Goodin (eds.) The Oxford Handbook of Public Policy. Oxford University Press, Oxford, pp. 689-708.

Mény, Y y Thoenig, J. C. (1992). Las políticas públicas. Ariel, Barcelona.

McCahery, J y Vermeulen, E. (2005). "Corporate Governance Crises and Related Party Transactions: A Post-Parmalat Agenda”, en Hopt, K., Wymeersch, E., Kanda, H., y Baum, H., (eds.). Corporate Governance in context. Oxford University Press, Oxford. 
Merton, R. (1999). "La estructura burocrática y la personalidad”, en Shafritz, J.M., y Hyde, A., (eds.). Clásicos de la administración pública, traducción de María Antonia Neira Bigorra. Colegio Nacional de Ciencias Políticas y Administración Pública, FCE, México.

Moore, M. (1995). Creating Public Value: Strategic Management in Government, Harvard University Press, Cambridge.

Offe, C. (2009). “Governance: an empty signifier?”, en Constellations, no 16, vol. 4, pp. 550562.

Olivo, M., Alaníz, C y Reyes, L. (2011). “Crítica a los conceptos de gobernabilidad y gobernanza: una discusión con referencia a los consejos escolares de participación social en México", en Revista Mexicana de Investigación Educativa, Vol. 16, número 50, julio/septiembre. México

Olsen, J. (2006). "Maybe it is time to rediscover bureaucracy", en Journal of Public Administration Research and Theory, $\mathrm{n}^{\circ} 16$, vol. 1, pp. 1-24

Olson, M. (1992). La lógica de la acción colectiva, Limusa-Noriega, México.

O'Toole Jr., L. (1997). The implications for democracy in a networked bureaucratic world, en Journal of Public Administration Research and Theory, nº 7, vol. 3, pp. 443-59.

Perry, J. Et al. (2014). "Returning to our roots: «good government» evolves to «good governance»", en Public Administration Review, n 74, vol. 1, pp. 27-28.

Peters, G. (1996). The Future of Governing: Four Emerging Models. Lawrence: University Press of Kansas.

Pierson, P. (2004). Politics in time: History, institutions, and social analysis. Princeton, University Press.

Prats, J. (2005). "La construcción social de la gobernanza", en Beltrán, J., y Prats, J. (coords.). Gobernanza: diálogo euro-iberoamericano sobre el buen gobierno. Ministerio de Administraciones Públicas (Colección: Estudio Goberna), Madrid.

Pressman, J y Wildavsky, A. (1984). Implementation: how great expectations in Washington are dashed in Oakland. University of California, Berkeley.

Rhodes, R. (1996). "The New Governance: Governing without Government”, en Political Studies, vol. xliv, pp. 652-667.

Rosanvallon, P. (2008). Counter democracy. Cambridge University Press, Cambridge.

Rothstein, B. (2011). The quality of government: corruption, social trust, and inequality in international perspective. Chicago University Press, Chicago.

Selznick, P. (1949). TVA and the Grass Roots: a Study in the Sociology of Formal Organization. University of California Press, Berkeley.

Shafritz, J y Hyde, A. eds. (1999). Clásicos de la administración pública, traducción María Antonia Neira Bigorra. Colegio Nacional de Ciencias Políticas y Administración Pública, FCE, México.

Shapiro, M. (2001). “Administrative Law Unbounded: Reflections on Government and Governance”, en Indiana Journal of Global, Legal Studies, nº 8, pp. 369-377.

Simon, H. (1947). Administrative behavior. University of Chicago Press. Chicago.

Simon, H., Smithburg, D y Thompson, V. (1991). Public administration. Transaction Publishers, New Brunswick.

Sørensen E y Torfing, J. (2005). “The democratic anchorage of governance networks", en Scandinavian Political Studies, no 28, vol. 3, pp. 195-218.

Sørensen, E y Torfing, J. (2003). «Network Politics, Political Capital and Democracy», en International Journal of Public Administration, número 26, pp. 609-34.

Walters, W. (2012). Governmentality. Critical encounters. Routledge. Londres

Weick, K. (2001). Making sense of the organization, Blackwell, Oxford.

Zingales, Luigi. (2008). "Corporate governance", en Blume, L. y Durlauf, S., (eds.). The new Palgrave Dictionary of Economics, second edition. 